\title{
Neuropalliative care
}

\section{Priorities to move the field forward}

Claire J. Creutzfeldt, MD, Benzi Kluger, MD, Adam G. Kelly, MD, Monica Lemmon, MD, David Y. Hwang, MD, Nicholas B. Galifianakis, MD, Alan Carver, MD, Maya Katz, MD, J. Randall Curtis, MD,

and Robert G. Holloway, MD

Neurology ${ }^{\circledR}$ 2018;91:217-226. doi:10.1212/WNL.0000000000005916

\section{Abstract}

Neuropalliative care is an emerging subspecialty in neurology and palliative care. On April 26, 2017, we convened a Neuropalliative Care Summit with national and international experts in the field to develop a clinical, educational, and research agenda to move the field forward. Clinical priorities included the need to develop and implement effective models to integrate palliative care into neurology and to develop and implement informative quality measures to evaluate and compare palliative approaches. Educational priorities included the need to improve the messaging of palliative care and to create standards for palliative care education for neurologists and neurology education for palliative specialists. Research priorities included the need to improve the evidence base across the entire research spectrum from early-stage interventional research to implementation science. Highest priority areas include focusing on outcomes important to patients and families, developing serious conversation triggers, and developing novel approaches to patient and family engagement, including improvements to decision quality. As we continue to make remarkable advances in the prevention, diagnosis, and treatment of neurologic illness, neurologists will face an increasing need to guide and support patients and families through complex choices involving immense uncertainty and intensely important outcomes of mind and body. This article outlines opportunities to improve the quality of care for all patients with neurologic illness and their families through a broad range of clinical, educational, and investigative efforts that include complex symptom management, communication skills, and models of care.

\section{Introduction}

The past few decades have seen remarkable progress in lessening the burden of neurologic disease, for example by reducing the number of relapses and delaying disability in multiple sclerosis, ${ }^{1}$ by reducing symptoms and prolonging independence in Parkinson disease, ${ }^{2}$ and by preventing and even reversing certain types of stroke. ${ }^{3,4}$ Despite this progress, 1 billion people across the globe have a neurologic illness, and more than 1 in 10 deaths are caused by neurologic disease. ${ }^{5}$ Moreover, most neurologic diseases remain incurable, shorten a person's lifespan, reduce time to dependence, diminish quality of life, and are associated with pain and other physical, psychological, and spiritual sources of suffering that are often difficult to control.

Palliative care is an approach to medical care for patients with serious illness that focuses on pain and symptom management, psychosocial and spiritual support, and effective communication to improve the quality of life of patients and their families and caregivers. Palliative care has seen a remarkable growth in the past decade, and the majority of US hospitals now have palliative care services. ${ }^{6}$ In addition, evidence continues to accumulate suggesting a benefit of

\section{Correspondence}

Dr. Creutzfeldt

clairejc@uw.edu

\section{Editorial}

Palliative care needs are everywhere: Where do we begin?

Page 201

\footnotetext{
From the Department of Neurology (C.J.C.), University of Washington, Harborview Medical Center, Seattle; Department of Neurology (B.K.), University of Colorado Anschutz Medical Center, Denver; Department of Neurology (A.G.K., R.G.H.), University of Rochester Medical Center, NY; Department of Pediatrics (M.L.), Division of Child Neurology, Duke University Hospital, Durham, NC; Division of Neurocritical Care and Emergency Neurology (D.Y.H.) and Center for Neuroepidemiology and Clinical Neurological Research (D.Y.H.), Yale School of Medicine, New Haven, CT; Department of Neurology (N.B.G., M.K.), University of California in San Francisco; Department of Neurology (A.C.), Memorial Sloan Kettering Cancer Center, New York, NY; and Cambia Palliative Care Center of Excellence (J.R.C.), University of Washington, Seattle.

Go to Neurology.org/N for full disclosures. Funding information and disclosures deemed relevant by the authors, if any, are provided at the end of the article.
} 


\section{Glossary}

AAHPM = American Academy of Hospice and Palliative Medicine; AAN = American Academy of Neurology; ABPN = American Board of Psychiatry and Neurology; ACGME = Accreditation Council for Graduate Medical Education; EHR = electronic health record; ICD-10 = International Classification of Diseases, Tenth Revision.

palliative care for various kinds of diseases including cancer and heart disease, ${ }^{7}$ and several neurologic illnesses. ${ }^{8-10}$

The "palliative care approach" describes the type of care the patient and family receive rather than the type of clinician providing this care. Therefore, it encompasses both primary palliative care (provided by the patients' primary medical team, including neurologic care) and specialist palliative care (provided by clinicians with subspecialty training in palliative care). ${ }^{11}$ Within this framework, we define a "neuropalliative care approach" as care that focuses on the specific needs of patients with neurologic illness and their families, including both primary and specialist palliative care. Neuropalliative care thus encompasses both an emerging subspecialty within neurology and a holistic approach to people who have neurologic illnesses that require a unique skill set, as suggested in table 1 .

The importance of palliative care for neurologic disease is increasingly recognized, and we are seeing a growing number of educational initiatives and practice guidelines ${ }^{12-15}$ that address palliative care within different neurology specialties. However, most such undertakings rely on a small evidence base. Now is the time to ask the question: "What are the top priorities for improving the outcomes of patients with serious neurologic illness and their families through a palliative care approach?"

To address this question and to set an agenda to develop the evidence needed to improve the quality of care that we provide these patients, we convened a group of neurology and palliative care experts during the 2017 American Academy of Neurology (AAN) meeting. The goal of this meeting was to develop a focused set of clinical, research, and educational priorities to move the field of neuropalliative care forward.

\section{Methods}

Participants were national and international experts in the fields of neurology and palliative care (listed in the acknowledgment section). Invitations were sent out to neurologists and trainees with known interest in the field, including those who were on a neuropalliative care listserve. The meeting, the "Neuropalliative Care Summit," was held for one half day during the AAN meeting on April 26, 2017. Given this venue, the majority of participants were physicians, with only a minority of representatives from other clinician groups such as social work, spiritual care, or nursing and no patient representatives. Meeting participants formed breakout groups focused on developing a clinical, educational, and research agenda for improving palliative care in neurology. Group meetings were followed by a plenary session during which a representative from each group presented the groups deliberations and incorporated feedback from all participants. This report presents a summary of the strategic priorities developed for neuropalliative care organized into the 3 categories of (1) clinical practice, (2) education, and (3) research. An executive summary of these priorities is shown in table 2.

\section{Results}

\section{Clinical practice}

The following priorities were identified in the clinical practice breakout group: (1) develop and implement effective models to integrate palliative care into neurology in- and outpatient care; (2) develop and implement informative quality measures to evaluate and compare palliative approaches to each other and to standard care; (3) better align financial and other incentives to promote patient-centered care; and (4) improve access to hospice care and update hospice criteria for neurologic disorders.

\section{Develop and implement effective models of integrating palliative care into neurology}

The Institute of Medicine recommends that all people with serious illness have access to skilled palliative care, ${ }^{16}$ and this recommendation is endorsed by the $\mathrm{AAN},{ }^{17}$ the American Stroke Association, ${ }^{12}$ American Heart Association, ${ }^{18}$ and the Neurocritical Care Society. ${ }^{13}$ Skilled palliative care includes both primary palliative care skills (including timely identification of palliative care needs and basic management of pain and other symptoms, as well as discussions around prognosis, goals of care, code status, and suffering) and specialist palliative care (including management of more complex physical, psychosocial, and spiritual suffering, conflict resolution regarding goals or treatment options, or assistance in addressing cases of potentially inappropriate care). ${ }^{11,19}$

Evidence on how best to integrate palliative care into neurology is limited. Several models exist, both in the inpatient (and critical care) $)^{20,21}$ and in the outpatient setting, ${ }^{9,22-25}$ and include:

A. A consultative model where palliative care specialists are consulted-the traditional "neurologic" treatment stays within the neurologists' practice and patients are referred to see a palliative care specialist separately.

B. An integrated model where a palliative care approach is shared simultaneously across primary providers and specialty teams. In the outpatient setting, this model may be realized through a multidisciplinary clinic where neurologists, 
Table 1 Suggested core palliative care skills for neurologists

(a) Identify common palliative care needs associated with specific neurologic disorders

(b) Detect and manage whole body pain

(c) Provide basic psychosocial and spiritual support

(d) Acquire communication skills including empathetic listening

(e) Effectively estimate and communicate prognosis and uncertainty

(f) Master shared decision-making for common preference sensitive decisions

(g) Master shared decision-making and support for patients and families around tragic choices

(h) Be aware of palliative care options of last resort

(i) Recognize and manage caregiver distress and needs

palliative or neuropalliative care specialists, and an interdisciplinary team cohabitate a clinic space. ${ }^{26,27}$ In the inpatient setting, palliative or neuropalliative care specialists would join discussions on rounds and family meetings are held with both neurology and palliative care teams together. C. A primary neuropalliative care model involving palliative care education and training for neurologists to provide neuropalliative care themselves. In the outpatient setting, this might also include training nonneurologists (e.g., primary care providers, geriatricians) who take care of patients with neurologic disease.

These models are not mutually exclusive, and optimal coverage of palliative care needs for neurology will involve a combination of these approaches. Different models will also be needed for different settings. For example, academic institutions with around-the-clock availability of consultants will need different models than community practices. Rural and other relatively underserved areas may benefit from telehealth and tele-education models to increase access to palliative care or neuropalliative care specialists. ${ }^{28}$

All of these approaches require that neurologists have basic palliative care skills, that palliative care specialists learn basic tenets of neurology, and that we encourage the development of triage systems for specialist palliative care consultations, both in the inpatient and outpatient setting. ${ }^{11,16,29}$

\section{Develop and implement quality measures to improve practice}

Considerable variation exists in life-sustaining and end-of-life care practices for patients with neurologic diseases. This variation results from differences in patients and surrogate preferences, as well as differences in how well clinicians practice shared decision-making, including communication about prognosis and eliciting preferences. Given recent data showing dramatic variations in hospital-level rates of "comfort measures only" (CMO) orders in patients with stroke, and limited documentation of patient preferences for life-limiting therapies in the medical record, considerable quality improvement opportunities exist. ${ }^{30-32}$
Some palliative care-oriented quality measures already exist in the neurology literature. These include screening measures around the domains of symptom management and advance care planning for certain diseases (for example, amyotrophic lateral sclerosis, ${ }^{33}$ dementia, ${ }^{34}$ Parkinson disease, ${ }^{35}$ and inpatient and emergency neurology $\left.{ }^{36}\right)$. As we recognize the need for a high-quality neuropalliative care approach to all patients with serious neurologic illness, additional domains need to be considered. For example, the recently published palliative and end-of-life measure set by the National Quality Forum ${ }^{37}$ includes measures within the following domains: comfortable dying, symptom screening, beliefs and documentation of values, care preferences, and treatment preferences. Such measures can be incorporated into innovative quality-improvement programs that can benchmark quality of care and build tools such as reminder alerts and care documentation requirements within the electronic health record (EHR). ${ }^{38,39}$ As an example, if the goal is to ensure that patients' and families' social and spiritual support needs are met, a quality-improvement program would involve developing a tool to help clinicians assess for relevant needs, educating clinicians in appropriate communication skills and other skills to meet those needs (either themselves or through appropriate use of other resources, such as chaplaincy), developing policies to ensure these conversations are taking place with the appropriate clinicians and within an appropriate time frame, and designing tools within the EHR to document and communicate that process. When possible, these measures should also be compatible with other national efforts for data-tracking in palliative care, such as Measuring What Matters from the American Academy of Hospice and Palliative Medicine (AAHPM) $){ }^{40}$ the Quality Data Collection Tool for Palliative Care, ${ }^{45}$ or Palliative Care Quality Network. ${ }^{41}$

\section{Better align financial and other incentives to promote patient-centered care}

Palliative care is by nature interdisciplinary and includes social work, spiritual care, nursing, and advanced nursing practice. The services provided by many of these clinicians are not reimbursed by insurance and instead tend to be funded through other means and justified through (1) the 
Table 2 Neuropalliative care: Executive summary of strategic priorities

\begin{abstract}
Clinical
To develop effective models to integrate palliative care into neurology, including consultative models, primary palliative care models, and an integrated comanagement approach to care. This will include the development of tele-medicine approaches to improve access to palliative care services for those who have barriers to receiving such care based on geography or disability.

To develop and implement specific quality measures that can be tracked and quantified across providers and sites of care. These include domains such as quality of dying, symptom screening, documentation values and goals of care, and care preferences. ${ }^{37}$ Combining patient-reported data with important palliative care components such as periodic serious illness conversations will help evaluate these components and their effect on patient outcomes.

To better align incentives to promote patient-centered care by advocating for payment reform and use of appropriate evaluation and management codes that recognize advance care planning, palliative care, and coordination of care.
\end{abstract}

To improve access to hospice care and update hospice criteria for neurologic disorders.

\title{
Education
}

To reduce the stigma of palliative care and to help clinicians, patients, families, and other stakeholders understand the advantages of timely palliative care to promote informed choices and improve quality of life.

To improve palliative care education for neurologists, including standardizing a core palliative care skill set and palliative care experience that all neurology trainees should master, as well as more specialized training specific to certain diseases and needs.

To improve access to neuropalliative care education for all providers. For neurologists and trainees, this includes (neuro-)palliative care fellowships, graduate courses in palliative care, and specific (neuro-)palliative care and communication workshops. More neuro-specific educational tools need to be available to palliative medicine specialists and other clinicians.

To incorporate education and testing within official training programs-including the ABPN, HPM, and ACGME-will be an important step in motivating implementation of an educational standard in neuropalliative care.

\section{Research}

To better understand the natural history of neurologic disease, not only as it relates to mortality but also as it relates to outcomes considered most important to patients and to their families. This goal includes investigating the processes leading to these outcomes, such as communication and treatment decisions and the delivery of "goal-concordant care."

To develop methods to help identify the needs of an individual patient, family, and situation and prompt certain conversations (including goals of care discussions), specialist consultations, or hospice referral. This includes improving the tools to prognosticate neurologic illness and communicate the information to loved ones and decision-makers.

To develop better interventions (e.g., drugs, devices, service delivery strategies, and behavioral interventions) to meet the needs of our patients and their families, to manage distressing symptoms, and to improve care while reducing unwanted burden and costs.

To better understand how people make decisions-especially how they partner with clinicians in making those decisions (shared decision-making), what decisional support they need, and how cognitive biases (e.g., the disability paradox, adaptation, framing), emotions, or prognostic uncertainty influence decision-making - and to develop additional tools and decision aids to embed into the clinical work flows to facilitate decision-making.

To find optimal ways of integrating palliative care into the care of patients with neurologic illness and their families across academic and community settings and to educate neurologists, trainees, and other clinicians about neuropalliative care.

Abbreviations: ABPN = American Board of Psychiatry and Neurology; ACGME = Accreditation Council for Graduate Medical Education; HPM = Hospice and Palliative Medicine.

importance of health care innovation and improved quality of care, and (2) the cost savings incurred by reducing the utilization and intensity of health care. Managed care organizations, such as Veterans Affairs Medical Centers, or government-run health systems, such as the UK National Health Services have been early adopters of palliative care-in part because the cost savings to the system, in addition to improvement in quality of care, can justify the costs of staffing. In fee-for-service models, financial support is more challenging and requires institutional support, research grants, and philanthropy. Without such support, it is difficult for neurologists to meet their clinical billing requirements through palliative care visits, given that insurance reimbursement will not adequately cover the time spent with patients during these more time-intensive appointments. Understanding how to optimally code clinic visits for insurance billing purposes is important. This includes using Current Procedural Terminology (CPT) billing modifiers for prolonged service (e.g., 99354) or advance care planning (99497 or 99498). ${ }^{42}$ In addition to using the ICD-10 code for the primary neurologic disease that a patient has, adding an ICD-10 code for palliative care encounter (Z51.5, was V66.7 in ICD-9) is important to better track these visits. More can be done, however, by federal and state agencies, payers, and health systems to develop better reimbursement policies that promote patient-centered care.

In addition to financial incentives, it is also important to align other types of incentives to facilitate integration of palliative care into care for patients with neurologic disorders. For example, having clinical leadership prioritize palliative care in clinical services and education will advance the uptake of high- 
quality palliative care. Acknowledging success in meeting palliative care needs for patients and families can highlight the importance of this care.

\section{Improve access to hospice care and update hospice criteria for neurologic disorders}

Summit participants raised several issues concerning hospice care for patients with neurologic illnesses. First, criteria for hospice eligibility are well developed for cancer patients but tend to be less appropriate or absent for chronic neurodegenerative diseases. ${ }^{43}$ Evidence-based hospice criteria for neurologic illnesses are needed. Second, participants raised concerns that hospice systems and providers may be less comfortable accepting patients with neurologic diagnoses and may benefit from neurology-specific training and materials, such as education about storming after severe acute brain injury or the inclusion of a Parkinson disease-specific hospice kit that does not include haloperidol. Finally, hospice standards vary regionally, and there may be a need for modifications based on local and regional regulations and resources.

\section{Education}

The priorities identified in the education breakout group were to (1) reduce the stigma of palliative care; (2) create standards for primary palliative care education for neurologists; (3) increase access to neuropalliative care education for all providers; and (4) incorporate palliative care education and testing within residency and fellowship training programs.

\section{Reducing the stigma of palliative care}

Given the historical development of palliative care from endof-life care, summit participants raised concern that there may be a misconception among clinicians and patients that palliative care is equivalent to end-of-life care and that its introduction indicates imminent death. Clinicians may view death and decline as a medical failure instead of embracing the inevitable as an expected and natural outcome. Similarly, clinicians and patients may perceive palliative care as an approach when there is "nothing left to do," when in fact an aggressive palliative care approach requires expertise, creativity, and a commitment to reduce suffering and improve the quality of life and the quality of dying. ${ }^{44}$

Neurologists frequently shy away from discussing the "p" (for palliative) or " $h$ " (for hospice) words with patients because they are afraid to diminish patients' hopes. The literature suggests that the contrary is true: that timely conversations about goals of care begun before the final weeks of life do not increase depression, can improve quality of life, and are associated with a better alignment of care with patients' preferences. ${ }^{7,45}$ The cultural stigma surrounding palliative care limits its accessibility. ${ }^{46,47}$ Some argue that continued palliative care education, mentorship, and leadership in medical school, residency, and fellowship settings will be the most effective way to shift the perception of palliative care among medical providers, while others recommend "rebranding" a new term. Some neuropalliative care clinics have chosen to name their clinics "Supportive Care," "Next
Step," or "Complex Symptom Management" clinics in the hopes that these terms may be culturally more acceptable.

\section{Creating a primary palliative care education curriculum for neurologists}

All neurologists, including residents and physicians in practice, should be able to provide primary palliative care to patients with serious neurologic illness ${ }^{48}$ (table 1 ). Specialized training is needed for neurologists focusing on neuropalliative care to gain command of more advanced palliative care skills and knowledge. Some neurologists may require training focused on the palliative care needs of their subspecialty populations (for example, movement disorders, neuro-oncology) or specialized settings (for example, intensive care units, outpatient clinics, or hospice), whereas others may be broadly interested in neuropalliative care as its own specialty encompassing multiple diseases and settings. These approaches all have their own merits and each suggest different needs in terms of educational paths and materials.

Primary neuropalliative care education should parallel established practices in palliative care education using examples from neurologic illnesses and emphasizing unique aspects of neurologic disease. Educational efforts should be guided by empiric needs assessments of target audiences to determine current perceptions (and misperceptions), knowledge gaps, attitudes, and self-perceived needs. Other skills may be needed by clinicians working in this field, which are not specific to palliative care but are needed for effective care, such as working in teams and preventing burnout. ${ }^{49}$

\section{Access to neuropalliative care education \\ Neurologists and neurology providers}

The American Board of Psychiatry and Neurology (ABPN) recognized Hospice and Palliative Medicine as a subspecialty with a qualifying examination in 2006. Since 2012, a 12-month Accreditation Council for Graduate Medical Education (ACGME) fellowship is required to become board-eligible in Hospice and Palliative Medicine. In 2017, there were 130 accredited fellowships available in the United States. In the same year, the ABPN Credentials Department counted 53 "diplomates in the subspecialty of Hospice and Palliative Medicine," among an overall 14,268 neurologists with active certificates (personal communication with $\mathrm{ABPN}$ and ABPN.com). This discrepancy highlights the shortage of neuropalliative care specialists.

Midcareer neurology clinicians have a variety of options to build their palliative care skills. These include comprehensive palliative care courses (e.g., the University of Washington Graduate Certificate Course in Palliative Care); master programs in palliative care offered by some graduate schools (e.g., University of Colorado); courses for building palliative care leadership (e.g., the Harvard Medical School Palliative Care Education and Practice course); online educational materials and training modules as offered by the Education in Palliative and End-of-Life Care program (epec.net); and courses and workshops that focus on specific skills, such as 
communication (e.g., VitalTalk). Other opportunities for palliative care education and certification for physicians and other health care providers can be found through the Center to Advance Palliative Care. ${ }^{6}$

The AAN and other neurology subspecialty societies are increasingly offering palliative care courses during their annual meetings. Partnerships with other clinical organizations (e.g., National Association of Social Workers) and patient advocacy groups (e.g., Alzheimer's Association, ALS Association, etc.) should be pursued to develop materials appropriate for other disease-specific populations.

Finally, with a growing interest for more formal training in neuropalliative care, there is momentum for building dedicated neuropalliative care fellowships or creating specialized neurology tracks within existing fellowships (e.g., University of Colorado starting in 2018).

\section{Palliative medicine specialists}

Palliative medicine specialists who interact with patients and families affected by neurologic disease-be it in inpatient, outpatient, or hospice settings - need to understand basic principles of care specific to neurologic disease, including the unique aspects around prognosis, prognostic uncertainty, and the effect of cognitive biases. ${ }^{50}$ They, too, may benefit from additional training, as described above. FAST FACTS is another online resource that was founded and is edited by faculty at the Medical College of Wisconsin and currently has more than 300 "facts" on palliative care issues relevant to a variety of illnesses (pcnow.org). There are currently few neurology entries and new submissions can be easily uploaded and approved. Other potential avenues to increase reach within the palliative medicine community include creating a special interest group for neurology at the AAHPM and creation of neurology training modules for the National Hospice and Palliative Care Organization.

\section{Other professionals}

Those who interact with patients with neurologic illnesses and/or their families include geriatricians, social workers, chaplains, physical therapists, speech language pathologists, pharmacists, and nurses. Resources for Palliative Care Education can be found through the Center to Advance Palliative Care (capc.org). Palliative care certification and credentialing are found through professional websites (for example, the North American Social Work Credentialing Center and the Hospice and Palliative Credentialing Center for nurses).

\section{Incorporate education and testing within official training programs}

Minimal competencies should be set for each clinician group to standardize education across settings and to guide the creation of test questions for boards and in-service training examinations; for example, for neurologists to know hospice guidelines and how to refer patients to hospice. Adding palliative care material to neurology board examinations and other requirements (e.g., quality metrics) will be an important step in motivating implementation. Also discussed was whether a requirement for direct palliative medicine exposure (e.g., a certain number of weeks on a palliative care service) should be required for all neurology residents. Lastly, our ongoing neuropalliative care working group is open to interested members and aims to coordinate efforts for the creation and dissemination of materials and to reduce redundant efforts.

To disseminate these efforts, buy-in will be required from residency program directors and national boards (e.g., $\mathrm{ABPN}$, Hospice and Palliative Medicine, ACGME). Research is urgently needed to define current training gaps and inform the development of relevant competencies.

\section{Research agenda}

The research working group identified urgent priorities within the following areas: (1) Epidemiology and Outcomes; (2) Needs Assessments; (3) Interventions (pharmacologic, technology, and behavioral); (4) Patient and Family Engagement; and (5) Implementation and Dissemination. The agenda below focuses on what needs to be investigated without prescribing how these investigations should be pursued. Advancing this agenda will require the incorporation of complementary research methodologies, including qualitative research, mixed methods, health services research, and implementation science. Unique data sources, inclusive of both large datasets (e.g., registries or claims data) and qualitative sources (e.g., interviews or focus groups), will add rigor and depth. Identifying training opportunities and facilitating pathways for those interested and motivated in learning various methodologic approaches should be priorities for our neuropalliative care community.

\section{Epidemiology and outcomes research}

To ensure the delivery of high-quality neuropalliative care, we need to better understand the natural history of neurologic disease, not only as it relates to mortality but also as it relates to outcomes considered most important to patients and to their families, including quality of life, spiritual and psychological well-being, social reintegration, and quality of death. In addition, we must understand the processes leading to these outcomes, including communication and treatment decisions and the delivery of "goal-concordant care"-a term increasingly used to express the aim of ensuring "that care provided matches closely with each individual's goals." This aspect is of particular importance in neurologic disease where (1) patients are often unable to express their goals themselves once they are sick, and (2) where a future health state, including the ability to adapt to it, can be extremely hard to predict or imagine ("affective forecasting"). Domains important for public health include measures of costs or burden of treatment, health care utilization, and access to care.

Of the variety of different outcomes important in neuropalliative care, some may require novel measures to be developed while others may be assessed with existing validated tools. 
1. Patient outcomes: For patients with certain neurologic disorders, assessments of cognitive and psychological outcomes are of particular importance, as opposed to a traditional focus on purely functional outcomes. ${ }^{54}$ Because many patients with severe neurologic disease may be nonverbal, or unable to understand the measurement tool, assessments of patient outcomes may require or rely on the input from proxies (family members or caretakers) and therefore need to be validated for proxy assessment. In addition, visual scales and other nonverbal alternatives should be further evaluated.

2. Family and caregiver outcomes: Family and surrogates of patients with neurologic illness are at high risk of poor health outcomes of their own, such as poorer overall selfrated health and increased rates of depression. ${ }^{55}$ The difficulty caregivers face when their loved one is affected by a neurologic disease, especially those that affect cognition, presents unique challenges.

3. Decision outcomes: One main focus of palliative care is to improve patient-centered treatment decisions through improved communication and patient engagement. Better tools are needed to rate the process of decisionmaking and the quality and aspects of a decision, to better attain goal-concordant care (also see the patient engagement innovations section below).

4. Public health outcomes: Measuring health care costs and health care utilization can assist us in uncovering factors that contribute to disproportionate costs and potentially unwanted treatments, especially at the end of life. In addition, we need to better understand the variability in treatment decisions across hospitals, geographic regions, or racial groups, and consider interventions to reduce unexplained variance. ${ }^{56}$ Research on health care disparities is needed as it relates to detecting disparities in access to and delivery of neuropalliative care, understanding the factors that underlie them, and intervening to eliminate them. ${ }^{57}$

\section{Needs assessments}

Palliative care needs range from pain and physical distress to psychological and existential suffering. Patients and families with palliative care needs are more likely to have poor outcomes and may benefit from certain neuropalliative care interventions. A variety of proposed methods to identify patients or needs were reviewed, which can facilitate primary or specialist palliative care in a timely manner.

1. Palliative care needs checklists can help target care to the needs of an individual patient, family, and situation and prompt certain conversations or specialist consultations. They can help identify patients or families at high risk of psychological morbidity or those receiving care discordant to their values. One example from the neuro-ICU is the SuPPOrTT checklist, ${ }^{58}$ in which clinicians screen for unmet needs during daily rounds by asking several simple questions regarding the presence of support needs, pain or other symptoms, concerns about prognosis or treatment, and clarity of goals of care. Studying this and/or related instruments may help identify the optimal settings for implementation.

2. Serious illness conversation triggers are objective signs and symptoms that can prompt providers to initiate or readdress conversations about goals of care, patient values, or end of life. More research is needed regarding how best to identify the appropriate time to approach goals-of-care situations for patients with diseases that have unpredictable natural histories.

3. Estimating prognosis: A patient's prognosis guides communication and decision-making. Discussion regarding the need for research in neuroprognostication centered on understanding the proper role of using population-based outcomes data and clinical scales in determining prognosis for individual patients. Many of the existing scales predict relatively crude measures of functional outcome, without an emphasis on outcomes that may be relevant to individual patient or family quality of life. Clinical scales and outcome prognostication tools that specifically address cognitive outcomes and other outcomes relevant to patient and family/ caregiver quality of life are needed.

4. Hospice eligibility criteria: As discussed above (in the section "improve access to hospice care and update hospice criteria for neurologic disorders”), criteria for hospice eligibility are well developed for patients with cancer but tend to be less applicable for severe acute brain diseases or for chronic neurodegenerative disease. ${ }^{43}$ Logical hospice criteria need to be developed that focus not only on the amount of time left for the patient but on their specific needs.

\section{Intervention research}

To better meet the needs of our patients and their families, we need to investigate the use of drugs, devices, and behavioral interventions. One particularly challenging area of research is end-of-life care, where evidence-based medicine is almost entirely absent.

1. Drugs: Heightened awareness of the excessive use of opioids in this country emphasizes the importance of therapeutic trials for improved pain and symptom control, including in advanced or terminal illness. At the same time, efforts to address the opioid epidemic should not interfere with appropriate use of opioids, especially for patients with terminal illness and pain requiring opioids. ${ }^{59}$ Medications for other disabling symptoms, ranging from agitation to fatigue, are also needed.

2. Devices and technology: The role of information technology in hospice and palliative care is gaining enthusiasm and includes projects such as telecommunication for home hospice patients and caregivers, such as "virtual visits," web-based conferences, worksheets and expert feedback, or specific use of the EHR, including 
triggers to consult a specialist or prompts for a serious illness conversation. The use of mobile technology to better collect real-time patient-level information is being tested in a variety of other clinical and research settings; translation of this approach to the neuropalliative care field should be investigated.

3. Behavioral interventions need to be explored across a broad range of disorders and settings, evaluating the effect of communication interventions (e.g., teaching communication skills to clinicians, promoting advance care planning); support interventions (e.g., pairing patients or families with a specially trained support nurse, bringing a chaplain to a clinic visit); or psychological interventions (e.g., providing patients or families with coping skills, teaching emotional resilience to clinicians).

\section{Patient/family engagement}

Patients with neurologic illness, their families, and their clinicians face multiple complex decisions over the course of the illness and at the end of life. Many patients lose decisionmaking capacity and their family members become their surrogate decision-makers. There was consensus that research is needed to better understand how people make decisionsespecially how they partner with clinicians in making those decisions (shared decision-making), what decisional support they need, and how cognitive biases (e.g., the disability paradox, adaptation, framing), emotions, or prognostic uncertainty influence decision-making. ${ }^{50}$ Aspects of decision-making that were discussed included the following:

1. Communicating prognosis: Research on the best practices for disclosing uncertain or poor neurologic prognosis to patients and families is a clear area of need. Given the prevalence of prognostic uncertainty in neurology, frameworks to discuss uncertainty are high priority. Of particular relevance to neuropalliative care is understanding best approaches to communicating with surrogate decision-makers. Prior research has shown that patient-surrogate agreement is lower for neurologic disorders than for medical conditions. ${ }^{60}$

2. Advance care planning and serious illness communication: Research is needed to better evaluate the advance care planning process in patients with neurologic disorders. Specific goals may include assessing the rate that neurology patients participate in advance care planning, how and when planning should be updated as disorders evolve, and whether advance planning is associated with more goal-concordant care.

3. Decision aids: Novel decision aids need to be developed for common goals-of-care situations in clinical neuroscience to better elicit preferences and more informed choices. These tools will need to be developed with clear triggers for use and applicability across different cultures.

\section{Implementation and dissemination research}

Implementation science is the systematic study of methods to promote the uptake and integration of research findings into routine health care. ${ }^{61}$ Improving palliative care for patients with serious neurologic illness will require research not just to demonstrate efficacy and effectiveness of interventions but also to efficiently implement and disseminate interventions that promote effective, high-quality palliative care across evolving health systems. This will require in-depth knowledge of best practice and the science of implementation and organizational change, including input from diverse stakeholders. Evaluation of clinical and educational efforts and models of palliative care delivery is needed as outlined above. As in all other areas of medicine, telehealth (tele-consultations, videoconferences, e-learning initiatives, and remote monitoring) is a promising way of getting high-quality subspecialty care to smaller, remote hospitals and clinics and even to patients' homes. Research is needed to identify ways to integrate these technological advances into medical care including how to reimburse for them.

\section{Conclusion}

Neuropalliative care is an emerging field with a bright future. As we continue to make remarkable advances in diagnosis and treatment, there will be an increasing need to guide patients and families with neurologic disease through complex choices involving immense uncertainty and intensely important outcomes of mind and body. We need to confront these challenges head-on and develop and execute plans to address the priorities within this nascent field. Only then will we deliver on our promise to patients and families to provide optimal care, a palliative care approach to care, from diagnosis to those final moments of lives well lived.

\section{Author contributions}

Dr. Creutzfeldt: study concept and design, acquisition of data, analysis and interpretation, critical revision of the manuscript for important intellectual content, study supervision. Dr. Kluger: study concept and design, acquisition of data, analysis and interpretation, critical revision of the manuscript for important intellectual content, study supervision. Dr. Kelly: acquisition of data, analysis and interpretation, critical revision of the manuscript for important intellectual content. Dr. Lemmon: acquisition of data, analysis and interpretation, critical revision of the manuscript for important intellectual content. Dr. Hwang: acquisition of data, analysis and interpretation, critical revision of the manuscript for important intellectual content. Dr. Galifianakis: acquisition of data, analysis and interpretation, critical revision of the manuscript for important intellectual content. Dr. Carver: acquisition of data, analysis and interpretation. Dr. Katz: acquisition of data, analysis and interpretation, critical revision of the manuscript for important intellectual content. Dr. Curtis: analysis and interpretation, critical revision of the manuscript for important intellectual content. Dr. Holloway: study concept and design, acquisition of data, analysis and interpretation, critical revision of the manuscript for important intellectual content, study supervision. 


\section{Acknowledgment}

The authors thank the Neuropalliative Care Summit 2017 Participants Jessica Baker (Partners HealthCare, Boston, MA); Kate Brizzi (Massachusetts General Hospital, Boston); Claudia Chou (Mayo Clinic, Rochester, MN); Tara Cook (University of Pittsburgh, PA); Farrah Daly (Goodwin House Palliative Care and Hospice, Alexandria, VA); Danny Estupinan (Mayo Clinic, Jacksonville, FL); Corey Fehnel (Beth Israel Deaconess Medical Center, Boston, MA); Laura Foster (Partners HealthCare, Boston, MA); James Grogan (Indiana University School of Medicine, Indianapolis); Ralf Jox (Ludwig-Maximilian University, Munich, Germany, and Lausanne University, Switzerland); Neha Kramer (Rush University Medical Center, Chicago, IL); Jerome Kurent (Medical University of South Carolina, Charleston); Judy Long (University of California in San Francisco); Mara Lugassy (MJHS, Albert Einstein College of Medicine, New York, NY); Jerome Phillips (Mercy Health, St. Mary's Hospital, Michigan State University); Jorge Risco (University of Rochester Medical Center, NY); Maisha Robinson (Mayo Clinic, Jacksonville, FL); Aimee Sato (Children's National Health System, Washington, DC); Akanksha Sharma (University of Washington, Seattle); Christopher Tarolli (University of Rochester Medical Center, NY); Lynne Taylor (University of Washington, Seattle); Lauren Treat (Children's Hospital Colorado, Denver); Ludo Vanopdenbosch (AZ Sint Jan Hospital, Brugge, Belgium); Anastasia Vishnevetsky (Partners Health System, Boston, MA); Neal Weisbrod (University of Rochester Medical Center, NY).

\section{Study funding}

No targeted funding reported.

\section{Disclosure}

C. Creutzfeldt receives funding from the NIH-National Institutes of Neurological Disease and Stroke (NINDS) (K23 NS099421). B. Kluger receives funding from the National Institutes of Nursing Research (NINR), NINDS, Patient Centered Outcome Research Institute (PCORI): Improving Healthcare Systems, NIH, and the Davis Phinney Foundation; he has served as an expert witness for Elite Medical Experts, Carlson \& Carlson, Chayet \& Danzo, and Elizabeth A. Kleger \& Associates; and he receives honorarium for speaking for the Davis Phinney Foundation. A. Kelly reports no disclosures relevant to the manuscript. M. Lemmon receives funding from the National Palliative Care Research Center Career Development Award. D. Hwang reports no disclosures relevant to the manuscript. N. Galifianakis received a philanthropic gift from Dorskind Family Foundation and receives research support from Boston Scientific Corporation, NIH, and PCORI. A. Carver reports no disclosures relevant to the manuscript. M. Katz receives funding from the Patient Centered Outcomes Research Institute and NIH-NINR (R01 NR016037). J. Curtis receives funding from the Cambia Health Foundation. R. Holloway receives funding from the NIH and NY State Department of Health. Go to Neurology.org/N for full disclosures.
Received February 2, 2018. Accepted in final form April 6, 2018.

\section{References}

1. Freedman MS. Present and emerging therapies for multiple sclerosis. Continuum 2013;19:968-991.

2. Morgan JC, Fox SH. Treating the motor symptoms of Parkinson disease. Continuum 2016;22:1064-1085.

3. Guzik A, Bushnell C. Stroke epidemiology and risk factor management. Continuum 2017;23:15-39.

4. Rabinstein AA. Treatment of acute ischemic stroke. Continuum 2017;23:62-81.

5. World Health Organization. Neurological disorders: public health challenges [online]. 2006 Available at: who.int/mental health/neurology/neurodiso/en/. Accessed January 9, 2018

6. Center to Advance Palliative Care. America's Care of Serious Illness [online]. 2015. Available at: reportcard.capc.org/. Accessed January 1, 2018.

7. Kavalieratos D, Corbelli J, Zhang D, et al. Association between palliative care and patient and caregiver outcomes: a systematic review and meta-analysis. JAMA 2016; 316:2104-2114

8. Higginson IJ, McCrone P, Hart SR, Burman R, Silber E, Edmonds PM. Is short-term palliative care cost-effective in multiple sclerosis? A randomized phase II trial. J Pain Symptom Manage 2009;38:816-826.

9. Miyasaki JM, Long J, Mancini D, et al. Palliative care for advanced Parkinson disease: an interdisciplinary clinic and new scale, the ESAS-PD. Parkinsonism Relat Disord 2012; 18(suppl 3):S6-S9.

10. Veronese S, Gallo G, Valle A, et al. Specialist palliative care improves the quality of life in advanced neurodegenerative disorders: NE-PAL, a pilot randomised controlled study. BMJ Support Palliat Care 2017;7:164-172.

11. Quill TE, Abernethy AP. Generalist plus specialist palliative care: creating a more sustainable model. N Engl J Med 2013;368:1173-1175.

12. Holloway RG, Arnold RM, Creutzfeldt CJ, et al. Palliative and end-of-life care in stroke: a statement for healthcare professionals from the American Heart Association/ American Stroke Association. Stroke 2014;45:1887-1916.

13. Frontera JA, Curtis JR, Nelson JE, et al. Integrating palliative care into the care of neurocritically ill patients: a report from the Improving Palliative Care in the ICU Project Advisory Board and the Center to Advance Palliative Care. Crit Care Med 2015;43:1964-1977.

14. Mitchell SL. Clinical practice: advanced dementia. N Engl J Med 2015;372: 2533-2540.

15. Miller RG, Jackson CE, Kasarskis EJ, et al. Practice parameter update: the care of the patient with amyotrophic lateral sclerosis: multidisciplinary care, symptom management, and cognitive/behavioral impairment (an evidence-based review): report of the Quality Standards Subcommittee of the American Academy of Neurology. Neurology 2009; 73:1227-1233.

16. Institute of Medicine of the National Academies. Dying in America: Improving Quality and Honoring Individual Preferences Near the End of Life. 1st ed. Washington, DC: National Academies Press; 2015.

17. Palliative care in neurology. The American Academy of Neurology Ethics and $\mathrm{Hu}-$ manities Subcommittee. Neurology 1996;46:870-872.

18. Braun LT, Grady KL, Kutner JS, et al. Palliative care and cardiovascular disease and stroke: a policy statement from the American Heart Association/American Stroke Association. Circulation 2016;134:e198-e225.

19. Bosslet GT, Pope TM, Rubenfeld GD, et al. An official ATS/AACN/ACCP/ESICM/ SCCM policy statement: responding to requests for potentially inappropriate treatments in intensive care units. Am J Respir Crit Care Med 2015;191:1318-1330.

20. Nelson JE, Bassett R, Boss RD, et al. Models for structuring a clinical initiative to enhance palliative care in the intensive care unit: a report from the IPAL-ICU project (improving palliative care in the ICU). Crit Care Med 2010;38: $1765-1772$.

21. Cox CE, Curtis JR. Using technology to create a more humanistic approach to integrating palliative care into the intensive care unit. Am J Respir Crit Care Med 2016;193:242-250.

22. Melvin TA. The primary care physician and palliative care. Prim Care 2001;28 239-249.

23. Hui D, Bruera E. Models of integration of oncology and palliative care. Ann Palliat Med 2015;4:89-98.

24. Gandesbery B, Dobbie K, Gorodeski EZ. Outpatient palliative cardiology service embedded within a heart failure clinic: experiences with an emerging model of care. Am J Hosp Palliat Care 2018;35:635-639.

25. Blackhall LJ. Amyotrophic lateral sclerosis and palliative care: where we are, and the road ahead. Muscle Nerve 2012;45:311-318.

26. Su KG, Carter JH, Tuck KK, et al. Palliative care for patients with Parkinson's disease: an interdisciplinary review and next step model. J Parkinsonism Restless Legs Syndr 2016;7:1-12.

27. Kluger BM, Fox S, Timmons S, et al. Palliative care and Parkinson's disease: meeting summary and recommendations for clinical research. Parkinsonism Relat Disord 2017;37:19-26.

28. Worster B, Swartz K. Telemedicine and palliative care: an increasing role in supportive oncology. Curr Oncol Rep 2017;19:37.

29. Creutzfeldt CJ, Holloway RG, Curtis JR. Palliative care: a core competency for stroke neurologists. Stroke 2015;46:2714-2719.

30. Prabhakaran S, Cox M, Lytle B, et al. Early transition to comfort measures only in acute stroke patients: analysis from the Get With The Guidelines-Stroke registry. Neurol Clin Pract 2017;7:194-204. 
31. Robinson MT, Vickrey BG, Holloway RG, et al. The lack of documentation of preferences in a cohort of adults who died after ischemic stroke. Neurology 2016;86: 2056-2062.

32. Singh T, Peters SR, Tirschwell DL, Creutzfeldt CJ. Palliative care for hospitalized patients with stroke: results from the 2010 to 2012 national inpatient sample. Stroke 2017;48:2534-2540.

33. Miller RG, Brooks BR, Swain-Eng RJ, et al. Quality improvement in neurology: amyotrophic lateral sclerosis quality measures: report of the Quality Measurement and Reporting Subcommittee of the American Academy of Neurology. Neurology 2013;81:2136-2140.

34. Sanders AE, Nininger J, Absher J, Bennett A, Shugarman S, Roca R. Quality improvement in neurology: dementia management quality measurement set update. Neurology 2017;88:1951-1957.

35. Factor SA, Bennett A, Hohler AD, Wang D, Miyasaki JM. Quality improvement in neurology: Parkinson disease update quality measurement set: executive summary. Neurology 2016;86:2278-2283.

36. Josephson SA, Ferro J, Cohen A, Webb A, Lee E, Vespa PM. Quality improvement in neurology: inpatient and emergency care quality measure set: executive summary. Neurology 2017;89:730-735.

37. National Quality Forum. Palliative and End-of-Life Care 2015-2016 [online]. 2017. Available at: qualityforum.org/Palliative_and_End-of-Life_Care_Project_2015-2016. aspx. Accessed January 8, 2018.

38. Curtis JR, Sathitratanacheewin S, Starks H, et al. Using electronic health records for quality measurement and accountability in care of the seriously ill: opportunities and challenges. J Palliat Med 2018;21:S52-S60.

39. Schenker Y, Arnold R. The next era of palliative care. JAMA 2015;314:1565-1566.

40. Dy SM, Kiley KB, Ast K, et al. Measuring what matters: top-ranked quality indicators for hospice and palliative care from the American Academy of Hospice and Palliative Medicine and Hospice and Palliative Nurses Association. J Pain Symptom Manage 2015;49:773-781.

41. Pantilat SZ, Marks AK, Bischoff KE, Bragg AR, O’Riordan DL. The palliative care quality network: improving the quality of caring. J Palliat Med 2017;20:862-868.

42. Centers for Medicare \& Medicaid Services. Frequently asked questions about billing the Physician Fee Schedule for advance care planning services [online]. 2016. Available at: cms.gov/Medicare/Medicare-Fee-for-Service-Payment/PhysicianFeeSched/Downloads/ FAQ-Advance-Care-Planning.pdf. Accessed July 17, 2017.

43. Centers for Medicare \& Medicaid Services. Hospice [online]. 2017. Available at: cms. gov/Medicare/Medicare-Fee-for-Service-Payment/Hospice/index.html. Accessed September 25, 2017.

44. Drury L, Baccari K, Fang A, Moller C, Nagus I. Providing intensive palliative care on an inpatient unit: a full-time job. J Adv Pract Oncol 2016;7:60-64.
45. Mack JW, Weeks JC, Wright AA, Block SD, Prigerson HG. End-of-life discussions, goal attainment, and distress at the end of life: predictors and outcomes of receipt of care consistent with preferences. J Clin Oncol 2010;28:1203-1208.

46. Fadul N, Elsayem A, Palmer JL, et al. Supportive versus palliative care: what's in a name? A survey of medical oncologists and midlevel providers at a comprehensive cancer center. Cancer 2009; 115:2013-2021.

47. Rhondali W, Burt S, Wittenberg-Lyles E, Bruera E, Dalal S. Medical oncologists perception of palliative care programs and the impact of name change to supportive care on communication with patients during the referral process: a qualitative study. Palliat Support Care 2013;11:397-404.

48. Creutzfeldt CJ, Robinson MT, Holloway RG. Neurologists as primary palliative care providers: communication and practice approaches. Neurol Clin Pract 2016;6:40-48.

49. Sigsbee B, Bernat JL. Physician burnout: a neurologic crisis. Neurology 2014;83: 2302-2306.

50. Creutzfeldt CJ, Holloway RG. Treatment decisions after severe stroke: uncertainty and biases. Stroke 2012;43:3405-3408.

51. Sanders JJ, Curtis JR, Tulsky JA. Achieving goal-concordant care: a conceptual model and approach to measuring serious illness communication and its impact. J Palliat Med 2018;21:S17-S27.

52. Dzau VJ, McClellan MB, McGinnis JM, et al. Vital directions for health and health care priorities from a National Academy of Medicine Initiative. JAMA 2017;317:1461-1470.

53. Turnbull AE, Hartog CS. Goal-concordant care in the ICU: a conceptual framework for future research. Intensive Care Med 2017;43:1847-1849.

54. Kapoor A, Lanctot KL, Bayley M, et al. "Good outcome" isn't good enough: cognitive impairment, depressive symptoms, and social restrictions in physically recovered stroke patients. Stroke 2017;48:1688-1690.

55. Harding R, Gao W, Jackson D, Pearson C, Murray J, Higginson IJ. Comparative analysis of informal caregiver burden in advanced cancer, dementia, and acquired brain injury. J Pain Symptom Manage 2015;50:445-452.

56. George BP, Kelly AG, Schneider EB, Holloway RG. Current practices in feeding tube placement for US acute ischemic stroke inpatients. Neurology 2014;83:874-882.

57. Kilbourne AM, Switzer G, Hyman K, Crowley-Matoka M, Fine MJ. Advancing health disparities research within the health care system: a conceptual framework. Am J Public Health 2006;96:2113-2121.

58. Creutzfeldt CJ, Engelberg RA, Healey L, et al. Palliative care needs in the neuro-ICU. Crit Care Med 2015;43:1677-1684.

59. Glod SA. The other victims of the opioid epidemic. N Engl J Med 2017;376:2101-2102.

60. Shalowitz DI, Garrett-Mayer E, Wendler D. The accuracy of surrogate decision makers: a systematic review. Arch Intern Med 2006;166:493-497.

61. Peters DH, Adam T, Alonge O, Agyepong IA, Tran N. Implementation research: what it is and how to do it. BMJ 2013;347:f6753.

\section{Did You Know...}

... you can browse by subspecialty topics on Neurology.org?

Go to: Neurology.org and click on "Topics" in the top navigation bar.

\section{Committed to Making a Difference: 2019 American Academy of Neurology Research Program}

The American Academy of Neurology (AAN) is committed to making a profound difference in the lives of researchers, in turn making a difference in the lives of patients with brain disease. The ambitious 2019 AAN Research Program offers opportunities ranging from $\$ 130,000$ to $\$ 450,000$ and designed for all types of research across all career levels and discovery stages. Pave your own pathway to patient care by applying for one of the opportunities by the October 1, 2018, deadline.

Visit AAN.com/view/ResearchProgram today. 